\title{
Use of expandable metal stents in the treatment of bronchial obstruction
}

\author{
A K SIMONDS, J D IRVING, S W CLARKE, R DICK \\ From the Departments of Thoracic Medicine and Radiology, Royal Free Hospital, London
}

ABSTRACT An expandable metal stent has been used to treat bronchial collapse due to polychondritis in one patient and extrinsic bronchial compression secondary to bronchogenic tumour in another.

Large airway obstruction may be due to intraluminal disease, stenosis, tracheobronchial collapse, or extrinsic compression. Surgical reconstruction of the diseased segment of an airway may be successful, ${ }^{\prime}$ and in some cases of intraluminal malignancy laser treatment restores airway patency. ${ }^{2}$ Tracheobronchial stents are an alternative, however, in patients with airway collapse, stenosis, and obstruction caused by extrinsic compression. ${ }^{3-5}$ Wallace and coworkers have described the modification of an expandable intravascular stent for use in the tracheobronchial tree. ${ }^{6}$ We have examined its clinical application in two patients with bronchial obstruction.

\section{Methods}

The stents (Cook Inc) comprise $0.46 \mathrm{~mm}$ stainless steel wire arranged in zigzag fashion to form a cylinder (fig 1). Various widths and single or double stent lengths are available. Hooks on the outer aspect of the stent are designed to become embedded in tracheobronchial mucosa and hence secure the device.

The stents are inserted in compressed form, loaded into a delivery catheter. In both cases described below the patienthad a general anaesthetic. After insertion of an oral endotracu cheal tube the site and extent of bronchial obstruction wers assessed by means of a fibreoptic bronchoscope. Unde fluoroscopic control a skin marker was placed on the chesi wall at the site of maximal airway obstruction. Balloon dilatation of the bronchus was carried out ${ }^{7}$ if extrinsig compression was found (patient 2). The delivery catheter waso then introduced with the help of radiographic screening and the stent was released from the catheter once aligned with the skin marker. Correct placement was confirmed with the fibreoptic bronchoscope. An alternative method of introduct tion would be to place the stent under direct vision through rigid bronchoscope.

\section{Case reports}

\section{CASE 1}

A 55 year old ex-smoker presented with shortness of breath and a non-productive cough. He had a mildly hyperinflated chest with no added respiratory sounds, normal hearbू sounds, and no evidence of peripheral cartilage abnormalit $\mathbb{B}$ or joint disease. The chest radiograph was normal. Pulmon ary function tests showed an $\mathrm{FEV}_{1} / \mathrm{FVC}$ of $1 \cdot 5 / 3.0$ litres with normal transfer coefficient and 1.71 of trapped gas. There was no improvement after a trial of terbutaline or pred nisolone $30 \mathrm{mg} /$ day for two weeks. The full blood count was. normal with an erythrocyte sedimentation rate of $3 \mathrm{~mm}$ in

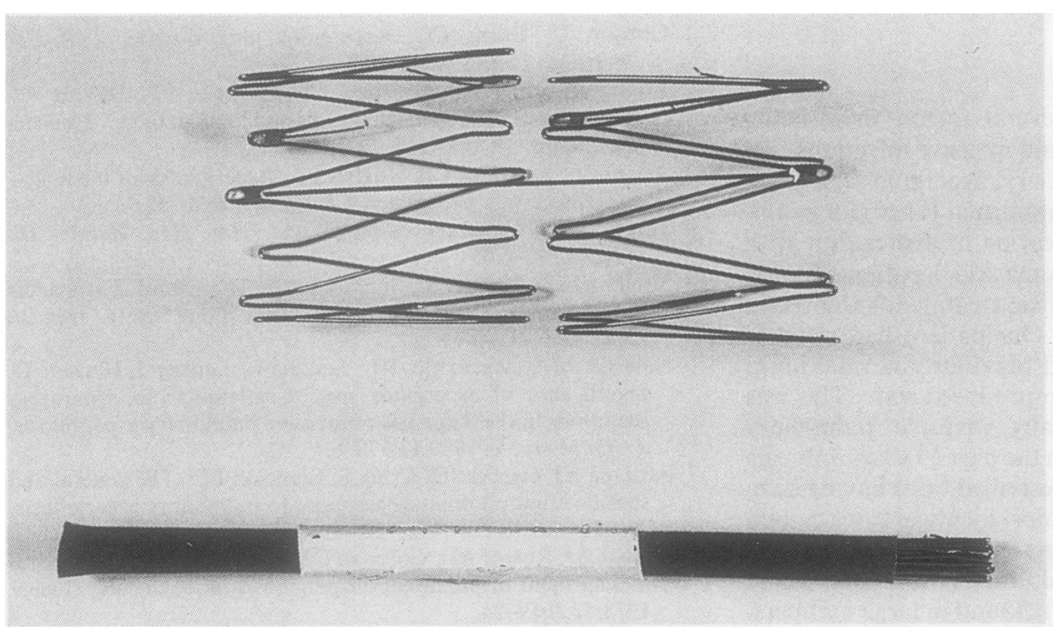

Fig 1 Expandable metal stent. 윽 Above: Double stent in expanded form. Below: Delivery catheter 을 containing compressed stent.

Address for reprint requests: Dr A K Simonds, Department of Thoracic Medicine, Royal Free Hospital, London NW3 2QG. 


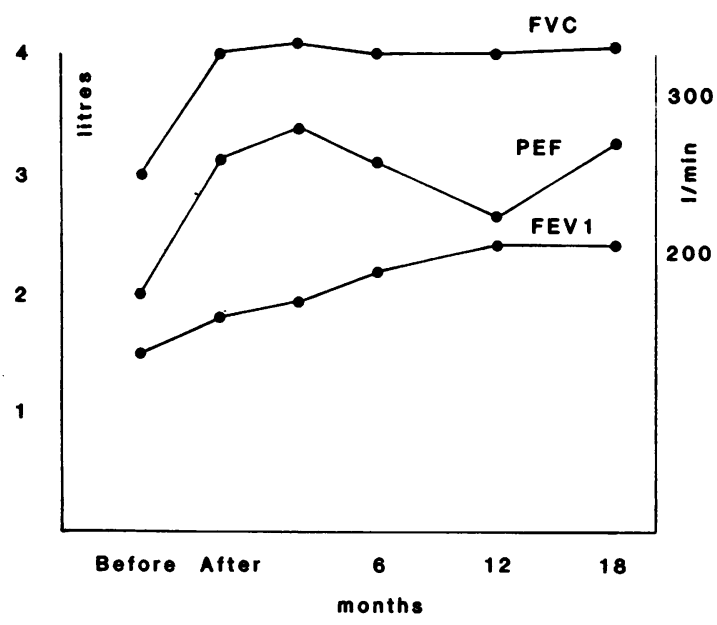

Fig 2 Case 1: Spirometry before and after insertion of stent.

one hour and negative findings in the autoantibody screen apart from a high titre of cartilage antibodies, consistent with a diagnosis of polychondritis. ${ }^{8}$ Rigid bronchoscopy showed a normal trachea and left main bronchus, but the right main bronchus was completely collapsed, opening only on forced inspiration. A diagnosis of probable polychondritis affecting the cartilage of the right main bronchus was made, and a 2.5 $\times 2 \mathrm{~cm}$ right endobronchial stent was inserted by the method described above. Within a day of insertion of the stent breathlessness had lessened. Serial spirometric values obtained over the next 18 months are shown in figure 2 . No adverse effects have been seen during follow up and chest radiographs have shown no change in the position of the stent.

CASE 2

A previously active 81 year old man presented with progressive shortness of breath of several months' duration. A squamous cell carcinoma of the right upper lobe had been diagnosed three years previously and treated with radiotherapy. On examination the trachea was deviated to the right with signs of fibrosis of the right upper lobe and monophonic wheeze in this region. Computed tomography showed recurrence of tumour with extrinsic compression of the right and left main bronchi by mediastinal lymphadenopathy. His peak expiratory flow (PEF) was $280 \mathrm{l} / \mathrm{min}$ and

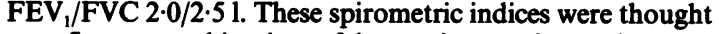
to reflect a combination of large airway obstruction and pulmonary fibrosis secondary to previous radiotherapy. The patient was treated by inserting two stents into the right main bronchus and one stent in the left main bronchus. A reduction in breathlessness was noted on recovery from the general anaesthetic, and his PEF had increased to $350 \mathrm{l} / \mathrm{min}$ and FEV $_{1} /$ FVC to $2 \cdot 6 / 3 \cdot 41$. Over seven months of follow up chest radiography has shown no alteration in the position of the stents. Symptomatic improvement was maintained and spirometric indices remained at values achieved after insertion of the stents for four months, enabling the patient to cope independently at home. He then became more breathless, with a fall in PEF to $300 \mathrm{l} / \mathrm{min}$ and in $F_{1} / F V C$ to
2.0/2.51. Computed tomography showed further mediastinal tumour spread with extension to the left hemithorax and encasement of left upper lobe. Tomography and fibreoptic bronchoscopy confirmed continued patency of the stents. Subsequent management has been on a symptomatic basis.

\section{Discussion}

Various stents have been used to maintain tracheobronchial patency. ${ }^{3-5}$ Expandable metal stents offer greater flexibility than silastic tube prostheses, a small surface area in contact with tracheobronchial mucosa, and minimal risk of occluding bronchial orifices if misplaced. Expandable stents should be considered only for permanent placement, however, as fixation of stent hooks to mucosal wall makes removal difficult. Experience with expandable metal stents placed in the airway has been limited to use in dogs and in patients with terminal disease. ${ }^{6}$ In dogs use of the stents was associated with the development of moderate cough. Neither of our patients developed cough or haemoptysis and there was no clinical evidence of bronchial inflammation in the present study.

Disease of the larnyx, trachea, and main bronchi occurs in more than half of patients with relapsing polychondritis and is a major cause of morbidity and mortality. ${ }^{10}$ Stenting may prove a useful adjunct to immunosuppression in this condition. Extrinsic tracheobronchial compression due to malignant disease is common and stents may provide important short term palliation of breathlessness. In both patients endobronchial placement of the expandable metal stent was technically simple and associated with no significant side effects. Further exploration of their clinical application in cases of permanent airway obstruction appears warranted.

\section{References}

1 Neville WE, Bolanowski PJP, Soltanzadeh H. Prosthetic reconstruction of the trachea and carina. $J$ Thorac Cardiovasc Surg 1976;72:525-38.

2 Hetzel MR, Nixon C, Edmonstone WM, et al. Laser therapy in 100 tracheobronchial tumours. Thorax 1985;40:341-5.

3 Montgomery WW. T-tube tracheal stent. Arch Otolaryngol 1965;82:320-1.

4 Westaby S, Jackson JW. A bifurcated silicone rubber stent for relief of tracheobronchial obstruction.J Thorac Cadiovasc Surg 1982;83:414-7.

5 Amemiya R, Matsushima Y, Kunii T, et al. Palliative tracheal tube stent without tracheotomy in tracheal stenosis. $J$ Thorac Cardiovasc Surg 1985;90:631-2.

6 Wallace MJ, Charnsagavej C, Ogawa K, et al. Tracheobronchial tree: expandable metallic stents used in experimental and clinical applications. Radiology 1986;158:309-12.

7 Cohen MD, Weber TR, Rao CC. Balloon dilatation of tracheal and bronchial stenosis. $A J R$ 1884;142:477-8.

8 Ebringer R, Rook G, Swana GT, Bottazzo GF, Doniach D. Autoantibodies to cartilage and type II collagen in relapsing polychondritis and other rheumatic diseases. Ann Rheum Dis 1981;40:473-9.

9 Michet CJ, McKenna CH, Luthra HS, O'Fallon WM. Relapsing polychondritis. Survival and predictive role of early disease manifestations. Ann Intern Med 1986;104:74-8.

10 Neilly JB, Winter JH, Stevenson RD. Progressive tracheobronchial polychondritis: need for early diagnosis. Thorax 1985;40:78-9. 